\title{
From dilute matter to the equilibrium point in the energy-density-functional theory
}

\author{
C.J. Yang, ${ }^{1}$ M. Grasso, ${ }^{1}$ and D. Lacroix ${ }^{1}$ \\ ${ }^{1}$ Institut de Physique Nucléaire, IN2P3-CNRS, Université Paris-Sud, F-91406 Orsay Cedex, France
}

\begin{abstract}
Due to the large value of the scattering length in nuclear systems, standard density-functional theories based on effective interactions usually fail to reproduce the nuclear Fermi liquid behavior both at very low densities and close to equilibrium. Guided on one side by the success of the Skyrme density functional and, on the other side, by resummation techniques used in Effective Field Theories for systems with large scattering lengths, a new energy-density functional is proposed. This functional, adjusted on microscopic calculations, reproduces the nuclear equations of state of neutron and symmetric matter at various densities. Furthermore, it provides reasonable saturation properties as well as an appropriate density dependence for the symmetry energy.
\end{abstract}

Various properties of nuclear matter are intimately related to nuclear phenomena. Such link strongly guides us in constraining effective interactions within the energydensity functional (EDF) theory, especially close to the equilibrium density of symmetric matter $\rho_{0}$, which roughly corresponds to central densities of medium-mass and heavy nuclei. Reproducing simultaneously the equation of state (EOS) of symmetric and pure neutron matter is an important step for producing interactions tailored to treat both stable and neutron-rich unstable nuclei or even, in the most extreme cases, the isospinasymmetric systems located in the crust of neutron stars. In phenomenological EDFs, attention is usually not paid to correctly describe the very low-density regime and only density scales of interest in nuclear phenomena are explored.

It is known that very dilute neutron matter can be described as an expansion in $k_{N} a$ given by Lee and Yang in Ref. 1], where $k_{N}$ is the neutron Fermi momentum and $a$ the neutron-neutron ${ }^{1} S_{0}$ scattering length, equal to $-18.9 \mathrm{fm}$. Such low-density regime is well described, by construction, in all $a b$-initio EOSs derived within effective field theories (EFTs) [2, 3]. To our knowledge, it is however never reproduced by phenomenological EOSs directly adjusted around the saturation point, such as, for instance, Skyrme [4, 5] or Gogny [6, 7] EOSs, even in those cases where a special care is taken in well describing the EOS of neutron matter [8, 9]

The first two terms of Lee-Yang expansion are

$\frac{E_{N M}}{N}=\frac{\hbar^{2} k_{N}^{2}}{2 m}\left[\frac{3}{5}+\frac{2}{3 \pi}\left(k_{N} a\right)+\frac{4}{35 \pi^{2}}(11-2 \ln 2)\left(k_{N} a\right)^{2}\right]$

where $m$ and $N$ are the nucleon mass and the number of neutrons, respectively, and the Fermi momentum is related to the density $\rho$ by $k_{N}=\left(3 \pi^{2} \rho\right)^{1 / 3}$. However, this expression is valid only in the very dilute regime, that is for $\left|a k_{N}\right|<<1$. In the nuclear case, owing to the large value of the scattering length, this limit corresponds to extremely low densities, definitely far from typical nuclear densities.

Recently, efforts were made to connect phenomenological EDFs and EFTs based on contact interactions [10]. Similarly to what is done in the many-body Dyson expansion [11], the total energy computed with EDF theo-

\begin{tabular}{cccc}
\hline$t_{0}\left(\mathrm{MeV} \mathrm{fm}^{3}\right)$ & $t_{3}\left(\mathrm{MeV} \mathrm{fm}^{4}\right)$ & $x_{0}$ & $x_{3}$ \\
\hline \hline-1803.93 & 12911.00 & -4.46 & -139.40 \\
\hline
\end{tabular}

TABLE I: Values of fitted parameters $x_{0}$ and $x_{3}$ and corresponding $t_{0}$ and $t_{3}$ values.

ries can be written as an expansion

$$
E\left(k_{N}\right)=E^{(1)}\left(k_{N}\right)+E^{(2)}\left(k_{N}\right)+\cdots,
$$

where the leading order $E^{(1)}$ is the mean-field-EDF (MF-EDF) energy and $E^{(2)}$ is the second-order perturbation energy treated with proper regularization. This approach was recently applied to obtain beyond-meanfield functionals reproducing nuclear matter properties [12, 13]. Within the EDF theory, we discuss here the possibility of combining an explicit $\rho$ dependence in an effective interaction with the constraint of correctly reproducing the very low-density regime, that is, of reproducing the first two terms of the Lee-Yang expansion. To make this, we first analyze symmetric and neutron matter with a simplified Skyrme interaction which contains the minimal terms for reproducing the saturation point at the leading order of the Dyson equation (MF approximation),

$$
v(\vec{r})=t_{0}\left(1+x_{0} P_{\sigma}\right) \delta(\vec{r})+\frac{1}{6} t_{3}\left(1+x_{3} P_{\sigma}\right) \rho^{\alpha} \delta(\vec{r}),
$$

where $P_{\sigma}=\frac{1}{2}\left(1+\sigma_{1} \cdot \sigma_{2}\right)$ is the spin-exchange operator, and $t_{0}, t_{3}, x_{0}, x_{3}$, and $\alpha$ are parameters.

The interaction given by Eq. (3) generates at each order of the Dyson equation a given density functional. From the MF density functional, one can deduce the MFEOS for neutron matter, that we report in terms of the Fermi momentum $k_{N}$,

$$
\begin{aligned}
\frac{E_{N M}^{(1)}}{N} & =\frac{3}{10} \frac{\hbar^{2}}{m} k_{N}^{2}+\frac{1}{12 \pi^{2}} t_{0}\left(1-x_{0}\right) k_{N}^{3} \\
& +\frac{1}{24} t_{3}\left(1-x_{3}\right)\left(\frac{1}{3 \pi^{2}}\right)^{\alpha+1} k_{N}^{3 \alpha+3}
\end{aligned}
$$

The term in $k_{N}^{3}$ in Eq. (1) has the same $k_{N}$ dependence as the contribution generated at leading order by the $t_{0}$ 
term of the interaction, with the relation

$$
t_{0}\left(1-x_{0}\right)=4 \pi \hbar^{2} a / m .
$$

It is indeed possible to mimic also the following term $\left(k_{N}^{4}\right)$ of the Lee-Yang expansion still using the density functional provided by the MF. One possibility would be to use the non-local interaction proposed in Ref. [14]. We follow here another direction and use the explicit density dependence of the effective interaction. The choice $\alpha=1 / 3$ leads to a $k_{N}^{4}$ term in the EOS (such direction was already explored for instance in Refs. [15, 16] in the case of dilute Fermi gases in the unitary regime). For $\alpha=1 / 3$, by comparing Eqs. (1) and (4), it must hold

$$
t_{3}\left(1-x_{3}\right)=\frac{\hbar^{2}}{m} \frac{144}{35}\left(3 \pi^{2}\right)^{1 / 3}(11-2 \ln 2) a^{2} .
$$

Eqs. (5) and (6) leave in this case only two free parameters in Eq. (3).

We write now the MF-EOS for symmetric matter in terms of the Fermi momentum $k_{F}\left(k_{F}=\left(3 \pi^{2} \rho / 2\right)^{1 / 3}\right)$,

$$
\frac{E_{S M}^{(1)}}{A}=\frac{3}{10} \frac{\hbar^{2}}{m} k_{F}^{2}+\frac{1}{4} \frac{t_{0}}{\pi^{2}} k_{F}^{3}+\frac{1}{16} t_{3}\left(\frac{2}{3 \pi^{2}}\right)^{\alpha+1} k_{F}^{3 \alpha+3},
$$

where $A$ indicates the number of nucleons. We adjust the remaining two free parameters of the interaction to reproduce a benchmark EOS for symmetric matter, that we have chosen as the SLy5-MF [8] EOS. This fit was successfully performed leading to the parameters listed in Table I. The incompressibility modulus associated to such EOS is $240.52 \mathrm{MeV}$. This adjustment is however a partial success. It indeed allows us to reproduce by construction the correct (very) low-density behavior in neutron matter and a reasonable saturation point in symmetric matter but, unfortunately, the resulting neutron matter EOS is completely wrong beyond the low-density limit. To obtain a satisfactory EOS for neutron matter at higher densities, the scattering length should be treated as a free parameter. However, by adjusting on a benchmark EOS for neutron matter, such fit would provide values of $a$ very far from $-18.9 \mathrm{fm}$ as noted in Ref. [10].

Along the line of the expansion given in Eq. (2), we then enrich the functional by adding to the MF EOSs the corresponding second-order contributions, calculated in Refs. [12, 13], by keeping only the finite parts (after dimensional regularization). For neutron matter, the second-order contribution is

$$
\frac{E_{N M}^{(2)}}{N}=c\left[t_{0}^{2}\left(1-x_{0}\right)^{2} k_{N}^{4}+f_{03} k_{N}^{3 \alpha+4}+f_{3} k_{N}^{6 \alpha+4}\right],
$$

where $c=m^{*}(11-2 \ln 2) /\left(280 \pi^{4} \hbar^{2}\right)$ and $m^{*}$ is the effective mass. Expressions for the coefficients $f_{03}$ and $f_{3}$ may be found in Ref. [13]. The second-order correction for neutron matter contains a $k_{N}^{4}$ contribution which is provided by the $t_{0}^{2}\left(1-x_{0}\right)^{2}$ term. Using the constraint of

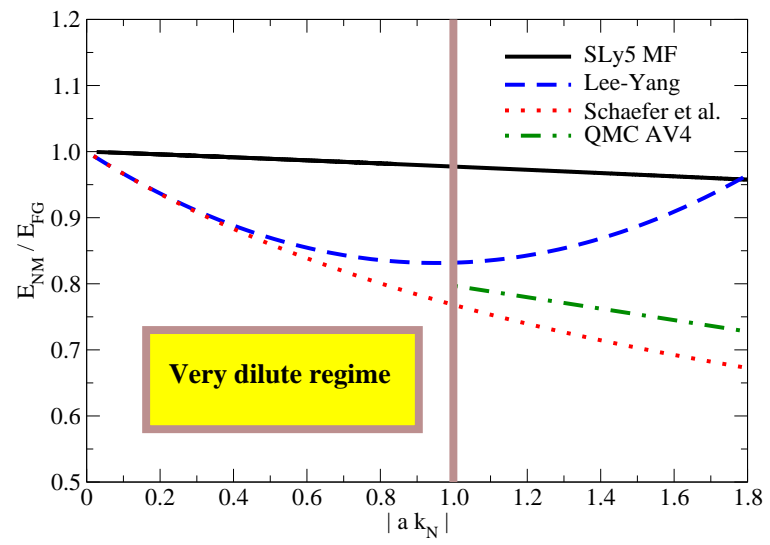

FIG. 1: (Color online) Neutron matter energy divided by the free gas energy $E_{F G}$, obtained with the first two terms of LeeYang formula, Eq. (1), (blue dashed line), a resumed formula of Ref. [3] (red dotted line), the SLy5-mean-field EOS (black solid line), and the QMC AV4 calculations of Ref. [19] (green dot-dashed line).

Eq. (5), one recovers the second term of the Lee-Yang expression, and could thus guarantee a correct low-density behavior. It is also interesting to observe that, to guarantee that the smallest $k_{N}$ dependences in the EOS are $k_{N}^{3}$ and $k_{N}^{4}$, as in the Lee-Yang expansion, the parameter $\alpha$ should be larger than $1 / 3$, which is not always the case for Skyrme forces. By using the second-order EOS for neutron matter, we have performed the adjustment of the Skyrme parameters on a benchmark EOS, keeping the additional constraint of reproducing the first two terms of the Lee-Yang expansion in the case of neutron matter. In spite of the correct $k_{N}$ dependence provided by the second-order contribution, it turns out that such fit is not successful, except in the case where the value of $a$ is kept free. However, this leads to an adjusted value of $a$ close to $-1 \mathrm{fm}$, definitely very far from the physical one. This direction was then rejected.

The first terms of the Lee-Yang expansion provide a correct behavior for neutron matter only for $\left|a k_{N}\right|<<1$. To produce expressions that are meaningful also at typical nuclear density scales, still keeping the good property of correctly reproducing the low-density regime, various resumed expressions have been proposed in EFT [3, 17, 18]. In its simplest form, a resumed formula may be for instance written as [3]

$$
\frac{E_{N M}}{N}=\frac{\hbar^{2} k_{N}^{2}}{2 m}\left[\frac{3}{5}+\frac{2}{3 \pi} \frac{k_{N} a}{1-6 k_{N} a(11-2 \ln 2) /(35 \pi)}\right] .
$$

In Fig. 1, the energy obtained with this expression is compared to Eq. (11) as well as to recent Quantum Monte Carlo (QMC) calculations [19] based on realistic nuclear forces [20]. The different curves are in agreement at very low densities. As an illustration, the Skyrme SLy5 MF EOS is also shown. It completely fails to reproduce the low density behavior. On the other side, Skyrme EDFs are recognized for reproducing remarkably well the equi- 


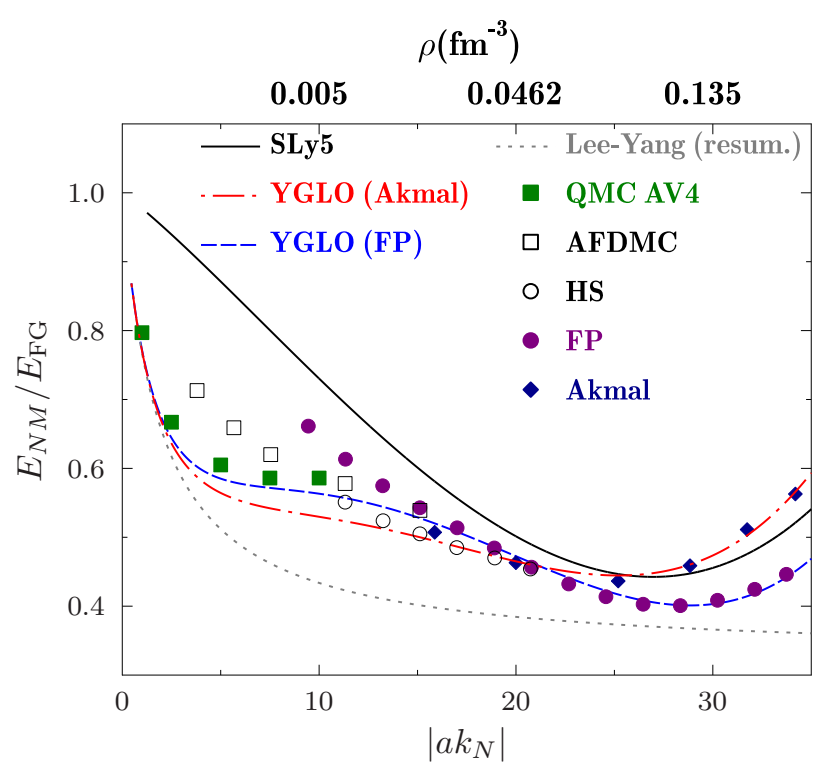

FIG. 2: (Color online) Energy of neutron matter divided by the free gas energy $E_{\mathrm{FG}}$ obtained with the two fits of this work, YGLO (FP) (blue dashed line) and YGLO (Akmal) (red dot-dashed line). The SLy5-MF EOS (black solid line) is also plotted together with the QMC AV4 points of Ref. [19] (green squares), the Friedman-Pandharipande (FP) results of Ref. 222] (violet circles), the Akmal et al. results of Ref. 23] (blue diamonds). The Auxiliary-Field results (AFDMC) of Ref. 28] and the N2LO calculation of Ref. 29] (HS) are also shown, respectively, with black open squares and black open circles. As an indication, 3 values of $\rho$ are provided in the upper horizontal axis.

librium features of symmetric matter and the incompressibility modulus. This is in particular due to the explicit two-body density-dependent term that is introduced in their expression.

Guided by the fact that the second-order $t_{0}$ contribution leads to the correct $k_{N}$ dependence in neutron matter (which can be associated to the second term of the Lee-Yang expansion), guided on one side by the resumed formulae of Refs. [3, 17, 18], and on the other side by the good properties of velocity-dependent and density-dependent terms in Skyrme forces, we propose a local density functional that includes resummation to all orders in an effective way. We write the energy as $E=\int\{\mathcal{K}(\mathbf{r})+\mathcal{V}(\mathbf{r})\} d^{3} \mathbf{r}$, where $\mathcal{K}$ is the kinetic term. The functional $\mathcal{V}$, to be used for symmetric $(\beta=1)$ and neutron $(\beta=0)$ matter, is given by

$$
\mathcal{V}=\frac{B_{\beta} \rho^{2}}{1-R_{\beta} \rho^{1 / 3}+C_{\beta} \rho^{2 / 3}}+D_{\beta} \rho^{8 / 3}+F_{\beta} \rho^{\alpha+2} .
$$

This functional leads to the following EOS,

$$
\frac{E}{A}=K_{\beta}+\frac{B_{\beta} \rho}{1-R_{\beta} \rho^{1 / 3}+C_{\beta} \rho^{2 / 3}}+D_{\beta} \rho^{5 / 3}+F_{\beta} \rho^{\alpha+1},
$$

where $A$ becomes $N$ in the case of neutron matter, and
$K$ is the kinetic contribution. We denote such functional with the acronym YGLO (for "Yang-Grasso-LacroixOrsay"). The two parameters $B_{\beta}$ and $R_{\beta}$ are fixed by imposing to recover the Lee-Yang formula at low density (the analog of Eq. (11) for symmetric matter may be found in Refs. [11, 21]). This gives the constraints

$$
B_{\beta}=2 \pi \frac{\hbar^{2}}{m} \frac{(\nu-1)}{\nu} a, R_{\beta}=\frac{6}{35 \pi}\left(\frac{6 \pi^{2}}{\nu}\right)^{\frac{1}{3}}(11-2 \ln 2) a
$$

where $\nu=2(4)$ is the degeneracy for $\beta=0$ (1), and $a$ is the corresponding scattering length. In principle, the scattering length used for symmetric matter should be an average over all the channels. Following the discussion of Ref. [11] (chapter XI), we take only the ${ }^{1} S_{0}$ scattering lengths and neglect the spin-triplet ${ }^{3} S_{1}$ neutron-proton contribution. This leads to an average ${ }^{1} S_{0}$ scattering lengths, $a \simeq-20 \mathrm{fm}$ for symmetric matter while for neutron matter we simply take $a=-18.9 \mathrm{fm}$.

The $C$-term in the denominator would provide (in the Taylor expansion) an additional higher-order contribution for the Lee-Yang expression. We have however preferred to keep such term free and use the coefficient as a parameter to adjust. We have then added explicitly other terms in the functional, guided by Skyrme-type forces, to correctly describe the EOSs at densities of interest in nuclear scales. The $\rho^{5 / 3}$ term mimics a term that would be produced (in a MF scheme) by a velocity-dependent zero-range interaction. In particular, such term turns out to be extremely important to improve the adjustment of the neutron matter EOS in density ranges between the very dilute regime and the saturation density. The $\rho^{\alpha+1}$ term in the EOS mimics a term that would be generated by a density-dependent two-body zero-range interaction like in Skyrme forces.

We perform the adjustments, this time using as benchmark microscopic EOSs, by including the constraints to describe the very low-density regime. Benchmark data are: i) For neutron matter, the QMC AV4 results of Ref. [19] for values of $\left|a k_{N}\right|<10\left(\rho<0.05 \mathrm{fm}^{-3}\right)$, and two different sets of results for $\left|a k_{N}\right|>10$ : the Friedman et al. results (FP) of Ref. 22] or the Akmal et al. results (stiffer EOS) of Ref. [23] [we call here the corresponding parameter sets YGLO (FP) and YGLO (Akmal), respectively]; ii) For symmetric matter, the FP results and those of Akmal et al. are very close from each others and we made a fit using only the FP points. The values of the YGLO parameters are shown in Table III.

Figure 2 shows the results obtained with the YGLO functional for neutron matter from the low-density regime to densities around saturation. This evolution is compared with the SLy5 MF curve together with several recent ab-initio calculations. We see that, with only four adjustable parameters, the new functional gives results in agreement with ab-initio calculations over the whole range of densities. In Fig. 3 the EOSs obtained for symmetric and neutron matter are shown as a function of $\rho$. In particular, one observes that the saturation properties 


\begin{tabular}{cccc}
\hline & $\begin{array}{c}C_{\beta} \\
\left(\mathrm{fm}^{2}\right)\end{array}$ & $\begin{array}{c}D_{\beta} \\
\left(\mathrm{MeV} \cdot \mathrm{fm}^{5}\right)\end{array}$ & $\begin{array}{c}F_{\beta} \\
\left(\mathrm{MeV} \cdot \mathrm{fm}^{3+3 \alpha}\right)\end{array}$ \\
\hline \hline$\beta=0(\mathrm{FP})$ & 100.87 & -9264.18 & 9571.90 \\
$\beta=0(\mathrm{Akmal})$ & 70.19 & -8377.83 & 8743.85 \\
$\beta=1(\mathrm{FP})$ & 8.188 & -6624.87 & 6995.46 \\
\hline
\end{tabular}

TABLE II: Values of the adjusted parameters obtained for the YGLO functional. In all cases, $\alpha=0.7$.

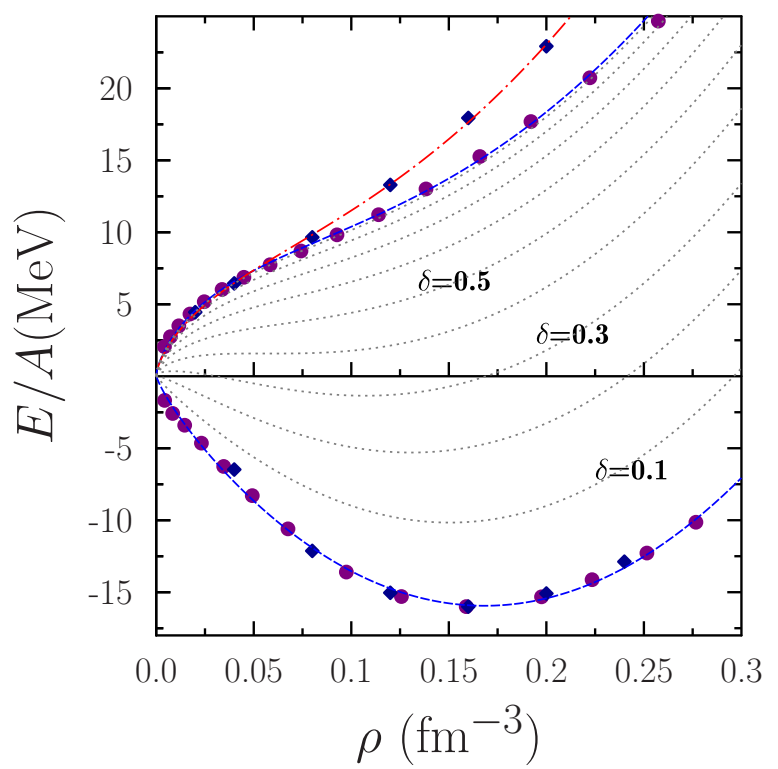

FIG. 3: EOSs from Akmal et al. 23] (blue diamonds) and Friedman et al. (purple circles) in symmetric and neutron matter compared to the YGLO (Akmal) (red dot-dashed curve) and YGLO (FP) (blue dashed curve) results. The different grey dotted curves correspond to the YGLO(FP) EOSs obtained for different asymmetry $\delta$ from 0.1 to 0.9 by steps of 0.1 (see text).

are well reproduced. The YGLO saturation density is $0.1683 \mathrm{fm}^{-3}$ and corresponds to an energy $E / A=-15.9$ $\mathrm{MeV}$. The incompressibility modulus is equal to 261.71 $\mathrm{MeV}$.

Starting from the two EOSs given above, one could use the standard parabolic approximation to obtain the EOS in asymmetric matter. Introducing the asymmetry parameter $\delta=\left(\rho_{N}-\rho_{P}\right) /\left(\rho_{N}+\rho_{P}\right)\left(\rho_{N}\right.$ and $\rho_{P}$ being the neutron and proton densities, respectively), the energy $E_{\delta}$ is given by

$$
\frac{E_{\delta}}{A}(\rho)=\frac{E_{S M}}{A}(\rho)+S(\rho) \delta^{2},
$$

where $S(\rho)$ is the symmetry energy, which can be computed, within the parabolic approximation, as the difference of the EOSs of neutron and symmetric matter. Corrections beyond such approximation are expected to be small [24, 25]. As an illustration, several EOSs obtained with different isospin asymmetries, from symmetric matter to pure neutron matter are displayed in Fig.

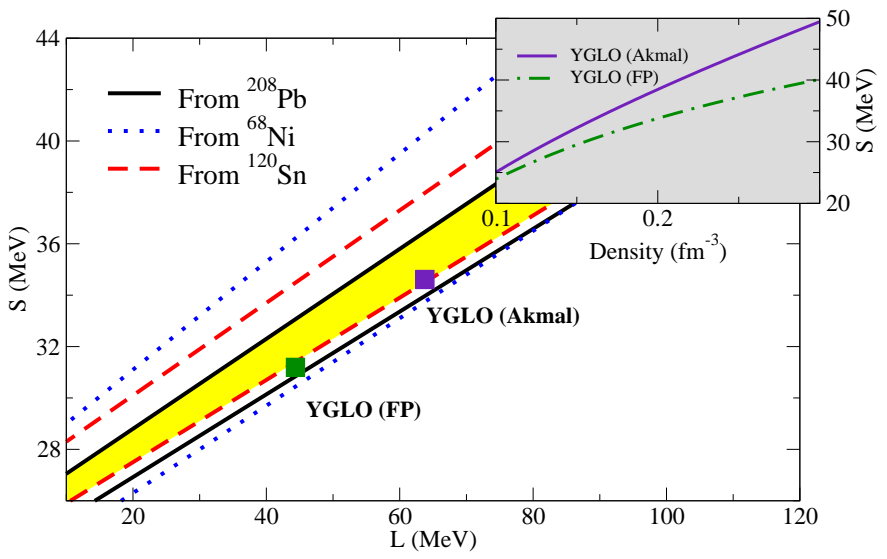

FIG. 4: Symmetry energy at saturation density as a function of its slope $L$. The black lines delimit the phenomenological area constrained by the experimental determination of the electric dipole polarizability in ${ }^{208} \mathrm{~Pb}$. The blue dotted lines delimit the area constrained by the same measurement in ${ }^{68} \mathrm{Ni}$, and the red dashed lines refer to the measurement done in ${ }^{120} \mathrm{Sn}$. The yellow area is the overlap. Inset: density dependence of the Symmetry energy for the two YGLO parametrizations of this work.

3 by employing the $\mathrm{YGLO}(\mathrm{FP})$ functional for neutron matter.

The density dependence of $S$ is known to be strongly connected to several nuclear properties of astrophysical interest such as, for instance, the proton fraction in neutron stars or to the thickness of neutron skins in neutronrich nuclei. In the inset of Fig. 4 the density dependences of $S$ are illustrated for the two YGLO parameterizations. These density dependences are comparable to those reported for instance in Ref. 26]. As expected, the YGLO parametrization adjusted on the Akmal et al. data provides a stiffer curve. A global quantity that characterizes the symmetry energy evolution around saturation is its slope $L=3 \rho_{0}(d S / d \rho)_{\rho=\rho_{0}}$. We present in Fig. 4 the points $(S, L)$ found in this work with respect to the phenomenological bands provided in Fig. 5 of Ref. [27]. These bands are dictated by the experimental determinations of the electric dipole polarizability in the nuclei ${ }^{208} \mathrm{~Pb},{ }^{68} \mathrm{Ni}$, and ${ }^{120} \mathrm{Sn}$. The yellow area is the overlap region of the three bands. We observe that both points are located at the lower limit of the yellow area. Note that many phenomenological EDFs are outside this band [27].

In the present work, inspired by resummation techniques used in EFT, we propose a local EDF that we call YGLO, able to describe the EOSs of symmetric and neutron matter from very low densities to the saturation density. We show that YGLO describes remarkably well saturation properties of symmetric matter, including incompressibility, and leads to a density dependence of the symmetry energy coherent with the phenomenological indications provided by the measurement of the dipole polarizability in nuclei. 
[1] T.D. Lee and C.N. Yang, Phys. Rev. 105, 1119 (1957).

[2] H.W. Hammer and R.J. Furnstahl, Nucl. Phys. A 678, 277 (2000)

[3] T. Schäfer, C.-W. Kao, and S.R. Cotanch, Nucl. Phys. A $762,82(2005)$.

[4] T.H.R. Skyrme, Philos. Mag. 1, 1043 (1956); Nucl. Phys. 9, 615 (1959).

[5] D. Vautherin and D. M. Brink, Phys. Rev. C 5, 626 (1972).

[6] D. Gogny, Nucl. Phys. A 237, 399 (1975).

[7] J. Dechargé and D. Gogny, Phys. Rev. C 21, 1568 (1980).

[8] E. Chabanat, P. Bonche, P. Haensel, J. Meyer, and R. Schaeffer, Nucl. Phys. A 627, 710 (1997); 635, 231 (1998); 643, 441 (1998).

[9] F. Chappert, N. Pillet, M. Girod, and J.-F. Berger, Phys. Rev. C 91, 034312 (2015).

[10] R.J. Furnstahl, Eft for DFT. " Renormalization Group and Effective Field Theory Approaches to Many-Body Systems. Springer Berlin Heidelberg, 2012. 133-191.

[11] A.L. Fetter and J.D. Walecka, Quantum Theory of Many-Particle Systems, (McGraw-Hill, New York, 1971).

[12] K. Moghrabi, M. Grasso, G. Colò, and N.V. Giai, Phys. Rev. Lett. 105, 262501 (2010).

[13] C.J. Yang, M. Grasso, X. Roca-Maza, G. Colò, and K. Moghrabi, arXiv:1604.06278 [nucl-th].

[14] A. Gezerlis and G.F. Bertsch, Phys. Rev. Lett. 105, 212501 (2010).
[15] A. Bulgac, Phys. Rev. A 76, 040502R (2007).

[16] A. Bulgac, M.M. Forbes, and P. Magierski, The Unitary Fermi Gas: From Monte Carlo to Density Functionals, Lecture Notes in Physics, Vol. 836 (Springer-Verlag, Berlin, Heidelberg, 2012), Chap. 9, pp. 305-373.

[17] J. V. Steele, arxiv:nucl-th/0010066 v2

[18] N. Kaiser, Nucl. Phys. A 860, 41 (2011).

[19] A. Gezerlis and J. Carlson, Phys. Rev. C 81, 025803 (2010).

[20] R. B. Wiringa and S. C. Pieper, Phys. Rev. Lett. 89, 182501 (2002).

[21] R. F. Bishop, Ann. Phys. 77, 106 (1973).

[22] B. Friedman and V. Pandharipande, Nucl. Phys. A361, 502 (1981).

[23] A. Akmal, V. R. Pandharipande, and D. G. Ravenhall Phys. Rev. C 58, 1804 (1998).

[24] W. Zuo, I. Bombaci, and U. Lombardo, Phys. Rev. C 60, 024605 (1999).

[25] W. Zuo, et al., Eur. Phys. J. A 14, 469 (2002).

[26] A.E.L. Dieperink, Y. Dewulf, D. Van Neck, M. Waroquier, and V. Rodin, Phys. Rev. C 68, 064307 (2003).

[27] X. Roca-Maza, et al., Phys. Rev. C 92, 064304 (2015).

[28] S. Gandolfi, A. Yu. Illarionov, S. Fantoni, F. Pederiva, and K. E. Schmidt, Phys. Rev. Lett. 101, 132501 (2008).

[29] K. Hebeler and A. Schwenk Phys. Rev. C 82, 014314 (2010). 